\title{
Desenvolvimento e comportamento larval de Leucochrysa (Leucochrysa) varia (Schneider) (Neuroptera, Chrysopidae) em laboratório
}

\author{
Euzileni Mantoanelli \& Gilberto S. Albuquerque
}

Laboratório de Entomologia e Fitopatologia, Centro de Ciências e Tecnologias Agropecuárias, Universidade Estadual do Norte Fluminense Darcy Ribeiro. Avenida Alberto Lamego 2000, 28013-602 Campos dos Goytacazes, Rio de Janeiro, Brasil. E-mail: gsa@uenf.br

\begin{abstract}
Development and larval behavior of Leucochrysa (Leucochrysa) varia (Schneider) (Neuroptera, Chrysopidae) in the laboratory. Laboratory studies with a predator commonly found in the Amazon and Atlantic forests of Brazil, Leucochrysa (Leucochrysa) varia (Schneider, 1851), revealed some characteristics that are atypical for green lacewings found in the tropics. First, its life cycle (egg to adult emergence) is relatively long, between $48\left(24^{\circ} \mathrm{C}\right)$ and 80 days $\left(18^{\circ} \mathrm{C}\right)$. Second, temperatures above $27^{\circ} \mathrm{C}$ are inappropriate for its development, since no specimen reached the adult stage under 27 and $30^{\circ} \mathrm{C}$ and linear relationship between developmental rate and temperature, for all stages, was only observed between 18 and $24^{\circ} \mathrm{C}$. Third, the lower thermal thresholds, between 7 and $10^{\circ} \mathrm{C}$, depending on the stage, is relatively low. Fourth, even though L. varia larvae are trashcarriers, they spend little of their time $(20 \%$ or less) loading detritus on their back, a proportion that apparently does not interfere with their developmental time. These results suggest that $L$. varia larvae probably explore microhabitats in the forest with temperatures much lower than those observed outside, and the large amount of time spent resting suggests that immobility is an important defense strategy, along with camouflaging.

KEY WORDS. Leucochrysini; life cycle; survival; temperature effect; trash-carrier.
\end{abstract}

RESUMO. Estudos em laboratório com Leucochrysa (Leucochrysa) varia (Schneider, 1851), predador comum nas florestas Amazônica e Atlântica do Brasil, revelaram algumas características atípicas para crisopídeos de regiões tropicais: 1) seu ciclo de vida (ovo à emergência do adulto) é relativamente longo, entre $48\left(24^{\circ} \mathrm{C}\right)$ e 80 dias $\left(18^{\circ} \mathrm{C}\right)$; 2$)$ temperaturas acima de $27^{\circ} \mathrm{C}$ não são apropriadas para seu desenvolvimento, já que nenhum espécime atingiu o estágio adulto a 27 e $30^{\circ} \mathrm{C}$ e só se observou relação linear entre taxa de desenvolvimento e temperatura, para todos os estágios, entre 18 e $24^{\circ} \mathrm{C}$; 3) os limiares térmicos inferiores, entre 7 e $10^{\circ} \mathrm{C}$, dependendo do estágio, são relativamente baixos; 4 ) apesar das larvas de $L$. varia serem carregadoras de lixo, elas despendem pouco de seu tempo (20\% ou menos) carregando detritos para seu dorso, proporção esta que aparentemente não interfere no seu tempo de desenvolvimento. Estes resultados sugerem que as larvas de $L$. varia provavelmente exploram micro-hábitats na floresta com temperaturas bem abaixo daquelas observadas fora dela e a grande parte do tempo que elas permanecem sem se mexer sugere que a imobilidade é uma importante estratégia de defesa, juntamente com a camuflagem. PALAVRAS-CHAVE. Bicho-lixeiro; ciclo de vida; efeito da temperatura; Leucochrysini; sobrevivência.

O gênero Leucochrysa McLachlan, 1868 é de longe o mais diverso da família Chrysopidae no mundo, com aproximadamente 190 espécies descritas e muitas outras aguardando descrição (Brooks \& Barnard 1990, Freitas \& Penny 2001). Está distribuído entre o norte dos Estados Unidos e a Argentina, com maior concentração de espécies nas regiões tropicais das Américas (PennY 1977, Penny et al. 1997, Freitas \& Penny 2001). Diferentemente de espécies pertencentes a outros gêneros de ocorrência neotropical mais estudados, como Chrysoperla Steinmann, 1964 e Ceraeochrysa Adams, 1982, que ocorrem em vegetações abertas, Leucochrysa spp. são típicas de bosques e florestas (ADAMs
1987, Freitas \& Penny 2001). Embora sejam predominantes tanto em diversidade como abundância nestes ecossistemas, além de terem se adaptado a agroecossistemas como pomares, seringais e eucaliptais, a biologia deste grupo de predadores ainda é praticamente desconhecida, assim como o papel que eles exercem no controle das populações de suas presas.

Um dos poucos aspectos biológicos estudados até o presente, e apenas superficialmente, é o comportamento de camuflagem. Como a maioria dos crisopídeos neotropicais, as larvas de Leucochrysa spp. se protegem contra seus inimigos naturais carregando um pacote de lixo sobre seu dorso, o qual 
é laboriosamente construído e constantemente reformado (Adams \& Penny 1987). Esta característica está intimamente associada com as cerdas de revestimento do corpo, que sustentam a carga de lixo (Gepp 1984). Enquanto as espécies cujas larvas são nuas, como as de Chrysoperla, apresentam cerdas pequenas, retas e esparsas, tanto no corpo como nos tubérculos laterais, as espécies com larvas carregadoras de lixo geralmente têm numerosas cerdas longas e com ponta em forma de gancho, especialmente no abdome e nos tubérculos laterais (Díaz-Aranda et al. 2001).

Os diferentes componentes do lixo carregado pelas larvas de várias espécies de Leucochrysa, como exoesqueletos de suas presas, exúvias de artrópodes, fibras de origem vegetal ou animal, ceras de insetos e outras partículas similares, são encontrados em troncos, galhos e folhas, onde elas procuram seu alimento (TAUber 2004). Em outros poucos casos, o pacote consiste em grande parte de líquens, como em Leucochrysa (Nodita) pavida (Hagen, 1861), cujas larvas formam associação simbiótica com estes organismos (Slocum \& LaWrey 1976). Nas duas situações, os pacotes de lixo são bem maiores do que aqueles observados em larvas carregadoras de lixo de outros gêneros neotropicais, como Ceraeochrysa e Chrysopodes Navás, 1923, embora o número de cerdas abdominais dorsais seja relativamente menor (TAUBER et al. 2000, Tauber 2003, 2004, Mantoanelli et al. 2006). Desta forma, seria esperado que larvas de Leucochrysa spp. alocassem um tempo maior para a construção e restauração do pacote de lixo, visando mantê-lo equilibrado sobre seu dorso relativamente desprovido de cerdas, o que diminuiria o tempo para a busca e captura de presas e comprometeria sua eficiência de crescimento. Para testar esta hipótese ainda não investigada, neste trabalho estudaram-se as características do desenvolvimento dos estágios imaturos e as atividades comportamentais das larvas de Leucochrysa (Leucochrysa) varia (Schneider, 1851), espécie abundante em fragmentos florestais remanescentes de Mata Atlântica nos Estados do Rio de Janeiro, Espírito Santo e Bahia e que também ocorre na Floresta Amazônica (G. S. Albuquerque, observações pessoais).

\section{MATERIAL E MÉTODOS}

\section{COLETA E CRIAÇÃo}

A criação de laboratório foi iniciada com fêmeas e machos de $L$. varia coletados com rede entomológica no interior de mata na Fazenda Babilônia ( $\left.21^{\circ} 51^{\prime} \mathrm{S}, 41^{\circ} 48^{\prime} \mathrm{W}\right)$, dentro dos limites do Parque Estadual do Desengano, remanescente de Mata Atlântica, em Campos dos Goytacazes, Rio de Janeiro. Estes adultos foram transferidos para o Laboratório de Entomologia e Fitopatologia da Universidade Estadual do Norte Fluminense Darcy Ribeiro (UENF), onde foram mantidos em gaiolas plásticas de $340 \mathrm{ml}$, com tampa telada na parte superior e tubo de vidro com água destilada, tampado com algodão, inserido em orifício na lateral. Os adultos foram alimentados com dieta à base de levedo de cerveja, frutose e mel (proporção de 1:1:1) e mantidos em câmaras de germinação do tipo BOD à temperatura de $24 \pm 1^{\circ} \mathrm{C}$ e fotoperíodo de 16 horas de luz: 8 horas de escuro.

Os estágios imaturos foram criados individualmente em tubos de ensaio de $40 \mathrm{ml}$, vedados com filme transparente de P.V.C. (Magipack ${ }^{\circledR}$ ), para evitar o canibalismo enquanto larvas. Estas foram alimentadas com ovos de Anagasta kuehniella (Zeller, 1879) (Lepidoptera, Pyralidae) durante todo o seu desenvolvimento. Após a mudança de estágio de pré-pupa para pupa, foi colocada uma tira de papelão dentro de cada tubo para auxiliar na fixação do adulto farado (estágio temporário após a saída do casulo, quando o adulto ainda está envolto pela cutícula da pupa), para que este pudesse efetuar a muda final para o estágio adulto propriamente dito. Os adultos foram identificados quanto ao sexo e transferidos para gaiolas iguais às descritas acima. Em cada gaiola, foi colocado um casal para obtenção de ovos e manutenção da criação. Exemplares adultos de L. varia provenientes da criação de laboratório encontram-se depositados na Coleção Entomológica da UENF.

\section{ProcedIMENTOS EXPERIMENTAIS}

Para estudar o desenvolvimento e sobrevivência dos estágios imaturos, o ensaio foi conduzido em delineamento inteiramente casualizado, com cinco tratamentos, correspondentes às temperaturas de $18,21,24,27$ e $30 \pm 1^{\circ} \mathrm{C}$, sob fotofase de 16 horas-luz/dia, em câmaras de germinação do tipo BOD. A umidade relativa no interior das câmaras variou entre 30 e 40\%, mas a real umidade percebida pelos estágios imaturos dentro dos tubos de ensaio fechados, maior devido à presença de dieta úmida, não foi medida. Sob estas condições, os insetos permaneceram desde o dia da oviposição até a emergência dos adultos. Foram alocados 25 ovos para cada tratamento, sendo feitas observações diárias a fim de determinar a duração dos estágios imaturos e a mortalidade em cada estágio. Os três instares do estágio larval foram considerados separadamente. Os estágios de pré-pupa e pupa foram agrupados para fins de análise devido à impossibilidade de observação do momento da mudança de estágio por transparência, pois os detritos que a larva de $L$. varia carrega em seu dorso são agregados ao casulo por ela tecido ao final de seu desenvolvimento.

Para quantificar os padrões de comportamento das larvas de $L$. varia, o ensaio experimental consistiu de observações das larvas em resposta aos ovos de A. kuehniella oferecidos, que serviram simultaneamente de material de camuflagem (ovos tanto intactos quanto sugados) e de alimento. Foram utilizadas 10 larvas provenientes da criação descrita acima, sendo que cada larva foi observada individualmente em duas idades durante o $1^{\circ}$ instar (chamadas de $1^{\circ}$ instar precoce e $1^{\circ}$ instar tardio) e $3^{\circ}$ instar ( $3^{\circ}$ instar precoce e $3^{\circ}$ instar tardio).

Com base em observações preliminares e também em estudo semelhante (Milbrath et al. 1993), cinco atividades distintas do comportamento larval foram selecionadas para quantificação: (1) alimentação: inserção de uma das mandíbulas no ovo, sucção de seu conteúdo interno e retração da mandíbula; (2) limpeza: esfregação das mandíbulas no substrato ou 
uma contra a outra; (3) carregamento (camuflagem): colocação ou rearranjo dos ovos sobre seu dorso; (4) imobilidade: ausência de movimento; (5) mobilidade: movimentos com a cabeça, corpo ou pernas, excluindo alimentação, limpeza e carregamento.

A arena experimental consistiu de uma pequena placa de Petri (3,5 cm de diâmetro) com um disco de papel filtro cobrindo o fundo, para facilitar o deslocamento das larvas, e aproximadamente $0,5 \mathrm{ml}$ de ovos de A. kuehniella, dispersos sobre todo esse disco. Cada período de observação durou 45 minutos, iniciado a partir da introdução da larva no centro da placa de Petri. Foi utilizado um microscópio estereoscópico para melhor visualização dos movimentos das larvas e um cronômetro para medir a duração de cada atividade em segundos. As atividades foram quantificadas na seqüência em que ocorriam, anotandose a sucessão de atividades e o tempo gasto em cada atividade. Também foram medidos o tempo usado pelas larvas de diferentes idades para consumir um ovo de A. kuehniella e o número total de ovos consumido no intervalo de 45 minutos. O $1^{\circ}$ instar precoce foi observado entre 4 e 11 horas após a eclosão, enquanto o $3^{\circ}$ instar precoce foi observado entre 6 e 16 horas após a muda ( $2^{\circ}$ para $3^{\circ}$ instar). Todas as larvas foram mantidas sem alimento/material de camuflagem da eclosão ou muda até o momento das observações. Após as observações, as larvas foram colocadas de volta em seus tubos e alimentadas por 54 horas. Após este período, o alimento e o pacote de lixo das larvas foram removidos, deixando-as nuas e sem alimento por um período de 12 horas antes da observação tardia de cada instar. Portanto, o $1^{\circ}$ instar tardio foi observado entre 70 e 77 horas após sua eclosão e o $3^{\circ}$ instar tardio entre 72 e 82 horas após a muda. A manutenção das larvas sem os ovos de $A$. kuehniella nos períodos citados acima, prévios às observações, serviu para assegurar que estas estariam mais propensas a desempenhar as atividades quantificadas, sem, entretanto, atingir um nível de estresse que afetasse seu comportamento normal.

\section{ANÁLISE ESTATÍSTICA}

A influência da temperatura sobre o desenvolvimento de L. varia foi expressa pela análise de regressão linear dos valores obtidos de cada estágio avaliado, correspondentes ao número de dias (d) para o desenvolvimento ( $1 / \mathrm{d}=$ taxa de desenvolvimento) em relação à temperatura. Além disto, foram calculados o limiar térmico inferior $(\mathrm{t})$, em ${ }^{\circ} \mathrm{C}$, e a constante térmica (K), expressa em graus-dia, para cada estágio e para todo o desenvolvimento (ovo - adulto). $\mathrm{O}$ valor de $\mathrm{t}$, correspondente à temperatura mínima abaixo da qual não há desenvolvimento, foi obtido pela extrapolação da linha de regressão sobre o eixo $\mathrm{x}$ (temperatura). $\mathrm{O}$ valor de $\mathrm{K}$, obtido pela fórmula $\mathrm{K}=(1 / \mathrm{y})(\mathrm{x}$ - t), onde $\mathrm{y}=$ taxa média de desenvolvimento e $\mathrm{x}=$ temperatura $\left({ }^{\circ} \mathrm{C}\right)$, representa o número de graus-dia, acima do limiar inferior, necessário para o inseto completar o desenvolvimento (WigGlesWORTH 1972).

Os resultados do comportamento das larvas relacionados à proporção de tempo alocado a cada atividade, tanto em relação ao total de 45 minutos como em relação a cada ocasião em que a atividade foi desempenhada, foram analisados através de ANOVA com dois fatores (instar e idade dentro de cada instar), usando-se o delineamento de blocos casualizados em esquema de sub-blocagem; as médias foram comparadas pelo teste de Tukey (pacote estatístico SAS). Para a comparação das médias de tempo requerido para o consumo dos ovos e do número de ovos predados, foi usado o teste $t$.

\section{RESULTADOS E DISCUSSÃO}

\section{DESENVOLVIMENTO E SOBREVIVÊNCIA DOS IMATUROS}

O tempo de desenvolvimento dos estágios imaturos de L. varia diminuiu com o aumento da temperatura (Tab. I). Entretanto, a $30^{\circ} \mathrm{C}$, observou-se que esta diminuição ficou aquém da esperada. Ao transformar-se os dados para taxas de desenvolvimento, as equações de regressão para cada um dos estágios, bem como os seus coeficientes de determinação $\left(\mathrm{R}^{2}\right)$, indicam que existe relação retilínea significativa entre as variáveis até $27^{\circ} \mathrm{C}$, excluindo-se o $3^{\circ}$ instar (retilínea de 18 a $24^{\circ} \mathrm{C}$ ), prépupa + pupa e desenvolvimento total (nenhum indivíduo completou o estágio de pupa a 27 e $30^{\circ} \mathrm{C}$ ) (Fig. 1). Os valores obtidos a $30^{\circ} \mathrm{C}$ não se enquadram na faixa de relação linear da taxa de desenvolvimento com a temperatura, o que sugere que temperaturas iguais ou superiores a $30^{\circ} \mathrm{C}$ estão fora da amplitude normal enfrentada por $L$. varia na natureza (WIGGLESWORTH 1972). Da mesma forma, o valor obtido para o $3^{\circ}$ instar a $27^{\circ} \mathrm{C}$ também sofreu um pequeno incremento no tempo de desenvolvimento em relação à $24^{\circ} \mathrm{C}$, vindo a sugerir que talvez esta temperatura esteja próxima do limite superior ótimo para o desenvolvimento desta espécie, conclusão que é corroborada pela mortalidade total dos indivíduos criados tanto a 27 como a $30^{\circ} \mathrm{C}$ antes de atingir o estágio adulto. Apesar de ser uma espécie de ocorrência tropical, L. varia só é encontrada no interior de florestas, onde as temperaturas são inferiores àquelas observadas exteriormente. Junto com sua capacidade de ocupar micro-hábitats mais apropriados ao desenvolvimento, estas características talvez expliquem o limiar superior de temperatura relativamente baixo desta espécie.

Se por um lado L. varia não suporta temperaturas muito elevadas, por outro esta espécie aparentemente pode se desenvolver a temperaturas mais baixas que outros crisopídeos, a julgar pelos limiares térmicos inferiores (t) obtidos para os diferentes estágios, entre 7,0 e $10,3^{\circ} \mathrm{C}$ (Tab. I). Para outras espécies, estes valores são relativamente maiores, como, por exemplo, as neotropicais Chrysopodes lineafrons Adams \& Penny, 1987 (9,9 a $12,3^{\circ} \mathrm{C}$ ) (Silva et al. 2007) e Chrysoperla externa (Hagen, 1861) (11,1 a $12,5^{\circ} \mathrm{C}$ ) (Albuquerque et al. 1994), assim como Chrysoperla carnea (Stephens, 1836) $\left(10,3\right.$ a 11,4 $\left.{ }^{\circ} \mathrm{C}\right)$, espécie holártica de clima temperado (TAuber \& TAUber 1976).

O tempo total de desenvolvimento (oviposição - emergência do adulto) variou entre aproximadamente 48 dias a $24^{\circ} \mathrm{C}$ e 80 dias a $18^{\circ} \mathrm{C}$. Nesta faixa de temperatura, outros crisopídeos já estudados apresentam desenvolvimento mais rápido. CANARD \& Principi (1984) e Canard \& VolKovich (2001), sumarizando a

Revista Brasileira de Zoologia 24 (2): 302-311, junho 2007 

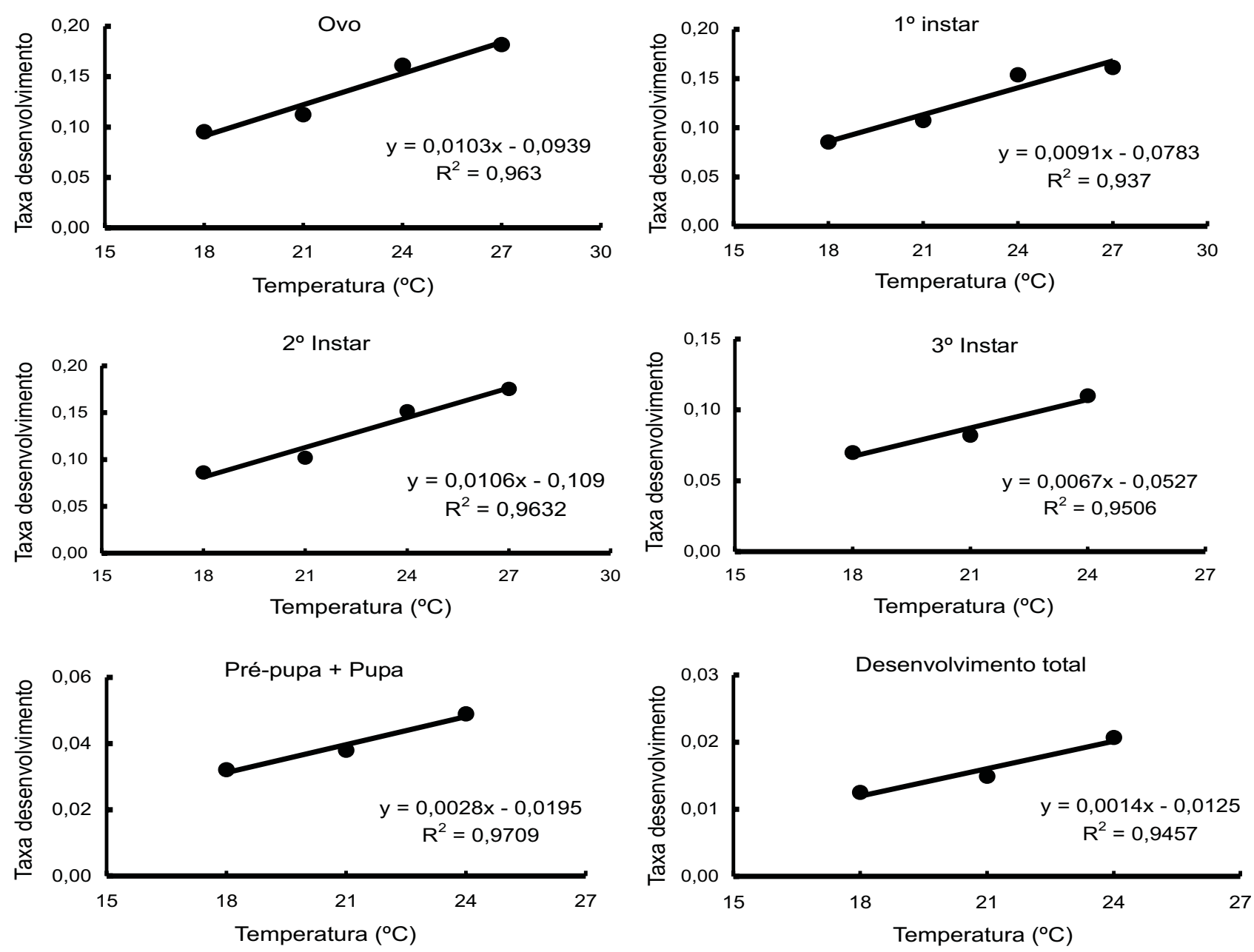

Figura 1. Relação entre taxas de desenvolvimento dos estágios de Leucochrysa varia e temperatura (fotoperíodo $=16$ horas luz: 8 horas escuro).

duração dos estágios imaturos de várias espécies de diferentes regiões do mundo, verificaram que a $24-25^{\circ} \mathrm{C}$ esta varia entre 24 e 35 dias para quase todas (11 espécies), com exceção de Chrysopodes placita (Banks, 1908), que leva 59 dias para completar seu desenvolvimento a $24^{\circ} \mathrm{C}$. Outros exemplos de espécies neotropicais de ambientes abertos indicam a mesma tendência: em C. lineafrons, a duração varia entre $31\left(25^{\circ} \mathrm{C}\right)$ e 64 dias $\left(18^{\circ} \mathrm{C}\right)$ (Silva et al. 2007), enquanto que em C. externa, esta varia entre $26\left(24^{\circ} \mathrm{C}\right)$ e 49 dias $\left(18^{\circ} \mathrm{C}\right)$ (Albuquerque et al. 1994). Portanto, em relação a outras espécies de crisopídeos conhecidas, pode-se concluir que o desenvolvimento de $L$. varia é bastante lento. Esta constatação é sustentada pela elevada constante térmica (K) para o desenvolvimento total obtida para $L$. varia, que foi de 750 graus-dia acima de $8,9^{\circ} \mathrm{C}$ (Tab. I). Este valor é bastante superior ao de outros crisopídeos, como C. externa (320 graus-dia) e C. lineafrons (470 graus-dia) (Albuquerque et al. 1994, Silva et al. 2007).
A sobrevivência dos estágios imaturos de $L$. varia foi baixa em todas as temperaturas, sendo que somente a 18, 21 e $24^{\circ} \mathrm{C}$ houve emergência de adultos (Tab. I). A maior sobrevivência, de $32 \%$, foi observada a $24^{\circ} \mathrm{C}$. Analisando-se separadamente por estágio, observou-se que no $1^{\circ}$ instar a sobrevivência foi sempre superior a $60 \%$, atingindo valores de até $96 \%$, enquanto no $2^{\circ}$ instar esta variou de 53 a $86 \%$ e, no $3^{\circ}$ instar, de 25 a 69\%, sendo que os menores valores de cada instar foram observados a $30^{\circ} \mathrm{C}$. Já no estágio de pré-pupa + pupa (dentro do casulo), a sobrevivência variou entre 56 e 73\%, exceto nas temperaturas de 27 e $30^{\circ} \mathrm{C}$, nas quais todos os indivíduos que sobreviveram até o estágio de pré-pupa + pupa morreram (Fig. 2). A sobrevivência relativamente baixa de L. varia pode ter sido devida à umidade relativa a que os imaturos foram expostos em laboratório, provavelmente inferior àquela que estes insetos enfrentam na natureza, pois vivem no interior de florestas tropicais úmidas. Alternativamente, pode ter resulta- 
Tabela I. Duração dos estágios de ovo, larva de $1^{\circ}, 2^{\circ}$ e $3^{\circ}$ instar, pré-pupa + pupa e total (ovo-adulto) e sobrevivência final sob cinco regimes de temperatura $\left(1^{\circ} \mathrm{C}\right.$; fotoperíodo $=16 \mathrm{~h}$ luz: $8 \mathrm{~h}$ escuro) e requerimentos térmicos para o desenvolvimento de Leucochrysa varia.

\begin{tabular}{|c|c|c|c|c|c|c|c|}
\hline \multirow{2}{*}{ Estágio } & \multicolumn{5}{|c|}{ Tempo de desenvolvimento ( $\bar{x} \pm \mathrm{EP}$, dias) } & \multicolumn{2}{|c|}{ Requerimentos térmicos ${ }^{a}$} \\
\hline & $18^{\circ} \mathrm{C}$ & $21^{\circ} \mathrm{C}$ & $24^{\circ} \mathrm{C}$ & $27^{\circ} \mathrm{C}$ & $30^{\circ} \mathrm{C}$ & $\mathrm{t}\left({ }^{\circ} \mathrm{C}\right)$ & K \\
\hline Ovo & $\begin{array}{c}10,5 \pm 0,15 \\
(n=25)\end{array}$ & $\begin{array}{c}8,9 \pm 0,13 \\
(n=25)\end{array}$ & $\begin{array}{c}6,2 \pm 0,07 \\
(n=25)\end{array}$ & $\begin{array}{c}5,5 \pm 0,10 \\
(n=25)\end{array}$ & $\begin{array}{c}5,0 \pm 0,00 \\
(n=25)\end{array}$ & 9,12 & 97,17 \\
\hline $1^{\circ}$ instar & $\begin{array}{c}11,7 \pm 0,18 \\
(n=24)\end{array}$ & $\begin{array}{c}9,3 \pm 0,17 \\
(n=22)\end{array}$ & $\begin{array}{c}6,5 \pm 0,12 \\
(n=24)\end{array}$ & $\begin{array}{c}6,2 \pm 0,15 \\
(n=21)\end{array}$ & $\begin{array}{c}6,1 \pm 0,15 \\
(n=15)\end{array}$ & 8,60 & 109,44 \\
\hline $2^{\circ}$ instar & $\begin{array}{c}11,6 \pm 0,22 \\
(n=19)\end{array}$ & $\begin{array}{c}9,8 \pm 0,34 \\
(n=13)\end{array}$ & $\begin{array}{c}6,6 \pm 0,13 \\
(n=16)\end{array}$ & $\begin{array}{c}5,7 \pm 0,19 \\
(n=18)\end{array}$ & $\begin{array}{c}5,9 \pm 0,23 \\
(n=8)\end{array}$ & 10,28 & 95,44 \\
\hline $3^{\circ}$ instar & $\begin{array}{c}14,3 \pm 0,50 \\
(n=9)\end{array}$ & $\begin{array}{c}12,2 \pm 1,50 \\
(n=5)\end{array}$ & $\begin{array}{c}9,1 \pm 0,39 \\
(n=11)\end{array}$ & $\begin{array}{c}9,7 \pm 0,50 \\
(n=9)\end{array}$ & $\begin{array}{c}9,0 \pm 2,01 \\
(n=2)\end{array}$ & 7,87 & 150,33 \\
\hline Pré-pupa + pupa & $\begin{array}{c}31,2 \pm 0,49 \\
(n=5)\end{array}$ & $\begin{aligned} 26,3 & \pm 0,34 \\
(n=3) & \end{aligned}$ & $\begin{aligned} 20,4 & \pm 0,26 \\
(n & =8)\end{aligned}$ & $\begin{array}{c}- \\
(n=0)\end{array}$ & $\begin{array}{c}- \\
(\mathrm{n}=0)\end{array}$ & 6,96 & 353,24 \\
\hline Desenv. total & $\begin{array}{c}79,8 \pm 1,09 \\
(n=5)\end{array}$ & $\begin{array}{c}67,0 \pm 1,00 \\
(n=3)\end{array}$ & $\begin{array}{c}48,3 \pm 0,89 \\
(n=8)\end{array}$ & - & - & 8,93 & 749,67 \\
\hline Sobrevivência (\%) & 20,0 & 12,0 & 32,0 & 0,0 & 0,0 & & \\
\hline
\end{tabular}

a foram excluídos, para efeito de cálculo, os valores obtidos a $30^{\circ} \mathrm{C}$ e, para o $3^{\circ}$ instar, também a $27^{\circ} \mathrm{C}$, por estarem fora da faixa de relação linear do desenvolvimento com a temperatura.

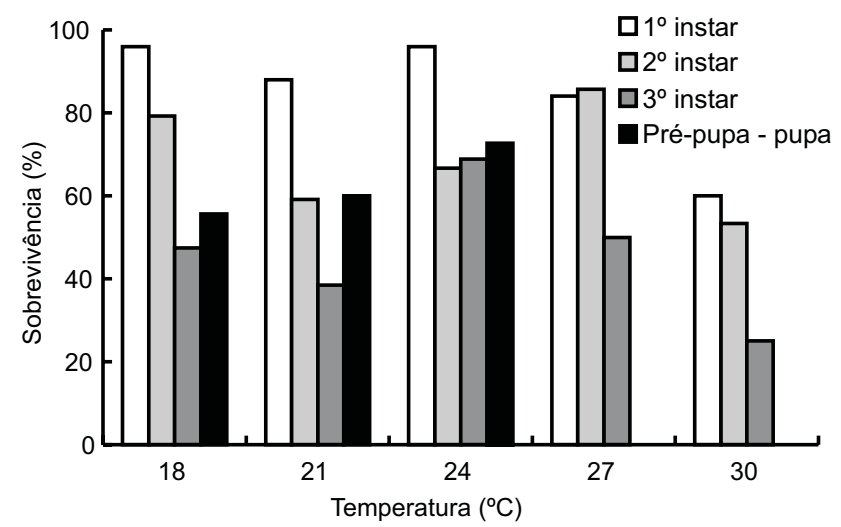

Figura 2. Sobrevivência dos três instares larvais e da pré-pupa + pupa de Leucochrysa varia, alimentados com ovos de Anagasta kuehniella, sob cinco regimes de temperatura constante $\left( \pm 1^{\circ} \mathrm{C}\right.$; fotoperíodo = 16 horas luz: 8 horas escuro).

do de algum problema nutricional, pois as larvas só receberam como alimento ovos de A. kuehniella, presa que não faz parte de sua dieta natural.

\section{COMPORTAMENTO DAS LARVAS}

No período de 45 minutos de observação, praticamente todas as larvas de $1^{\circ}$ e $3^{\circ}$ instar, tanto precoces como tardias, desempenharam, ao menos uma vez, todas os cinco atividades comportamentais previamente estabelecidas, isto é, alimentação, camuflagem, limpeza, mobilidade e imobilidade. Somente duas larvas precoces e três tardias de $1^{\circ}$ instar e três tardias de $3^{\circ}$ instar deixaram de executar entre uma e três dessas atividades. Também se observou que não existem seqüências estereotipadas de ações, pois, em geral, cada atividade foi seguida por qualquer outra; algumas seqüências, porém, ocorreram com maior probabilidade (Fig. 3). A proporção de tempo gasto em cada uma das atividades (no cômputo geral e em cada momento) variou tanto em relação ao instar quanto em relação à idade dentro de cada instar, embora nem sempre significativamente (Fig. 3, Tabs II e III).

\section{DESCRIÇÃO DAS ATIVIDADES DAS LARVAS Alimentação}

As larvas ingeriram o fluido dos ovos de A. kuehniella por sucção por meio de suas mandíbulas. Normalmente só uma mandíbula (esquerda ou direita, sem aparente preferência) foi inserida no ovo por vez; a outra mandíbula serviu para manipulá-lo, facilitando o movimento de vaivém da mandíbula suctora para atingir todo o interior do ovo. Após sugar todo o fluido, a larva retirou sua mandíbula do cório restante, abandonando-o ou transferindo-o para seu dorso.

Com exceção do $3^{\circ}$ instar tardio, onde foi a segunda atividade mais realizada, a alimentação foi preponderante em todas as idades observadas; as larvas alocaram entre 32 e 50\% de seu tempo a esta atividade (Fig. 3, Tab. II). Enquanto as proporções observadas nas duas idades do $1^{\circ}$ instar foram semelhantes, larvas tardias de $3^{\circ}$ instar despenderam tempo significativamente menor para alimentação que as precoces (Tab. II). Não houve diferença significativa nestas proporções entre os instares. Adicionalmente, em cada ocasião que se alimentavam, o tempo médio despendido pelas larvas de $1^{\circ}$ instar, de cerca de quatro minutos, foi significativamente superior àquele despendido pelas larvas de $3^{\circ}$ instar, de pouco mais de um minuto (Tab. III). Não houve diferenças significativas dentro de cada instar. Por outro lado, as larvas de $3^{\circ}$ instar repetiram a atividade de alimentação três vezes mais que as de $1^{\circ}$ instar no período de 45 minutos (Tab. II). 

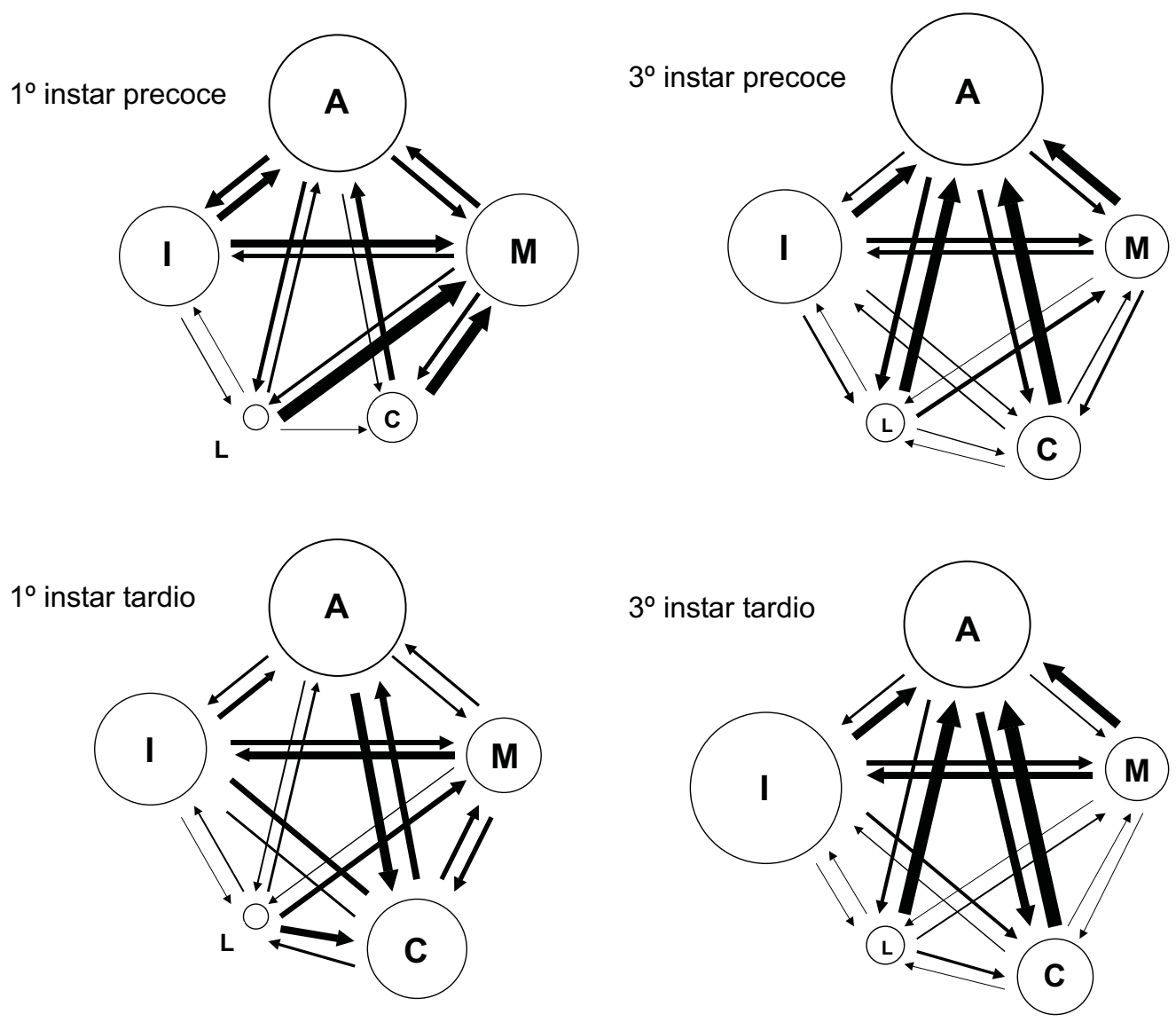

Figura 3. Etogramas do comportamento larval de Leucochrysa varia, em arena contendo ovos de Anagasta kuehniella, durante 45 minutos de observação $(n=10$ larvas/etograma). A área de cada círculo reflete a proporção média de tempo despendido em cada atividade: alimentação (A), camuflagem (C), mobilidade (M), limpeza (L) e imobilidade (I). A largura das flechas representa a probabilidade média de mudança da atividade de onde elas partem para a atividade onde elas apontam.

Tabela II. Proporções de tempo alocado às diferentes atividades pelas larvas de Leucochrysa varia frente à presa/material de camuflagem oferecido (ovos de Anagasta kuehniella), durante o período de 45 minutos de observação (média \pm EP; $\mathrm{n}=10$ larvas/instar/ocasião). Entre parênteses, número médio de vezes que as larvas repetiram a atividade no período de observação; número total médio de atividades/45 minutos = 22,9 ( $1^{\circ}$ instar precoce); 19,8 ( $1^{\circ}$ instar tardio); 40,7 ( $3^{\circ}$ instar precoce) e 36,9 ( $3^{\circ}$ instar tardio). (A) Significância das comparações de cada atividade comportamental entre idades no $1^{\circ}$ e no $3^{\circ}$ instar; (B) significância das comparações entre os instares. ${ }^{*}=\mathrm{p}>0,05{ }^{* *}=\mathrm{p}>0,01 ;{ }^{* * *}=\mathrm{p}>0,001 ; \mathrm{NS}=$ não significativo (ANOVA).

\begin{tabular}{|c|c|c|c|c|c|c|c|}
\hline \multirow{2}{*}{ Atividade } & \multicolumn{3}{|c|}{$1^{\circ}$ instar } & \multirow{2}{*}{ B } & \multicolumn{3}{|c|}{$3^{\circ}$ instar } \\
\hline & Precoce & $A$ & Tardio & & Precoce & $A$ & Tardio \\
\hline \multirow[t]{2}{*}{ Alimentação } & $0,391 \pm 0,053$ & NS & $0,404 \pm 0,036$ & NS & $0,500 \pm 0,044$ & * & $0,316 \pm 0,049$ \\
\hline & $(5,7)$ & & $(4,5)$ & & $(15,8)$ & & $(14,9)$ \\
\hline \multirow[t]{2}{*}{ Imobilidade } & $0,251 \pm 0,059$ & NS & $0,263 \pm 0,070$ & * & $0,295 \pm 0,035$ & * & $0,502 \pm 0,082$ \\
\hline & $(3,7)$ & & $(3,7)$ & & $(5,6)$ & & $(5,3)$ \\
\hline \multirow[t]{2}{*}{ Mobilidade } & $0,301 \pm 0,085$ & * & $0,117 \pm 0,042$ & $\star *$ & $0,084 \pm 0,024$ & NS & $0,068 \pm 0,023$ \\
\hline & $(7,8)$ & & $(3,5)$ & & $(7,0)$ & & $(3,4)$ \\
\hline \multirow[t]{2}{*}{ Camuflagem } & $0,044 \pm 0,013$ & $* * *$ & $0,200 \pm 0,048$ & NS & $0,082 \pm 0,020$ & NS & $0,096 \pm 0,023$ \\
\hline & $(2,3)$ & & $(6,0)$ & & $(5,5)$ & & $(9,1)$ \\
\hline \multirow[t]{2}{*}{ Limpeza } & $0,013 \pm 0,003$ & NS & $0,015 \pm 0,005$ & $* *$ & $0,039 \pm 0,005$ & $* *$ & $0,019 \pm 0,009$ \\
\hline & $(3,4)$ & & $(2,1)$ & & $(6,8)$ & & $(4,2)$ \\
\hline
\end{tabular}


Após cada ocasião de alimentação, as larvas realizaram qualquer uma das quatro outras atividades (Fig. 3). As larvas de $1^{\circ}$ instar precoce priorizaram a imobilidade (35\%) após a alimentação, enquanto as larvas de $1^{\circ}$ instar tardio priorizaram a camuflagem $(55,6 \%)$. No $3^{\circ}$ instar precoce, a limpeza $(35,4 \%)$ foi a principal atividade desempenhada após a alimentação, enquanto no $3^{\circ}$ instar tardio a camuflagem voltou a ser a prioridade $(50,3 \%)$. Portanto, nem sempre o ato de comer foi seguido pela limpeza das mandíbulas, como seria esperado.

A quantidade de tempo requerida para consumir um ovo de A. kuehniella variou em função do instar e, no caso do $1^{\circ}$ instar, também da idade (Fig. 4). O $1^{\circ}$ instar gastou cerca de qua- tro a seis vezes mais tempo que o $3^{\circ}$ instar. No $1^{\circ}$ instar, larvas tardias ingeriram o conteúdo do ovo mais rapidamente que as precoces, enquanto no $3^{\circ}$ instar não houve diferença significativa nos tempos de consumo das larvas precoces e tardias.

Em relação ao número médio de ovos predados no intervalo de 45 minutos, verificou-se que o $3^{\circ}$ instar consumiu três a sete vezes mais ovos que o $1^{\circ}$ instar (Fig. 5). Larvas de $1^{\circ}$ instar tardias comeram cerca de oito ovos neste intervalo, número significativamente maior que aquele consumido pelas precoces (cinco ovos). Para as larvas de $3^{\circ}$ instar, a tendência foi inversa; as precoces comeram um número significativamente maior que as tardias (37 contra 26 ovos).
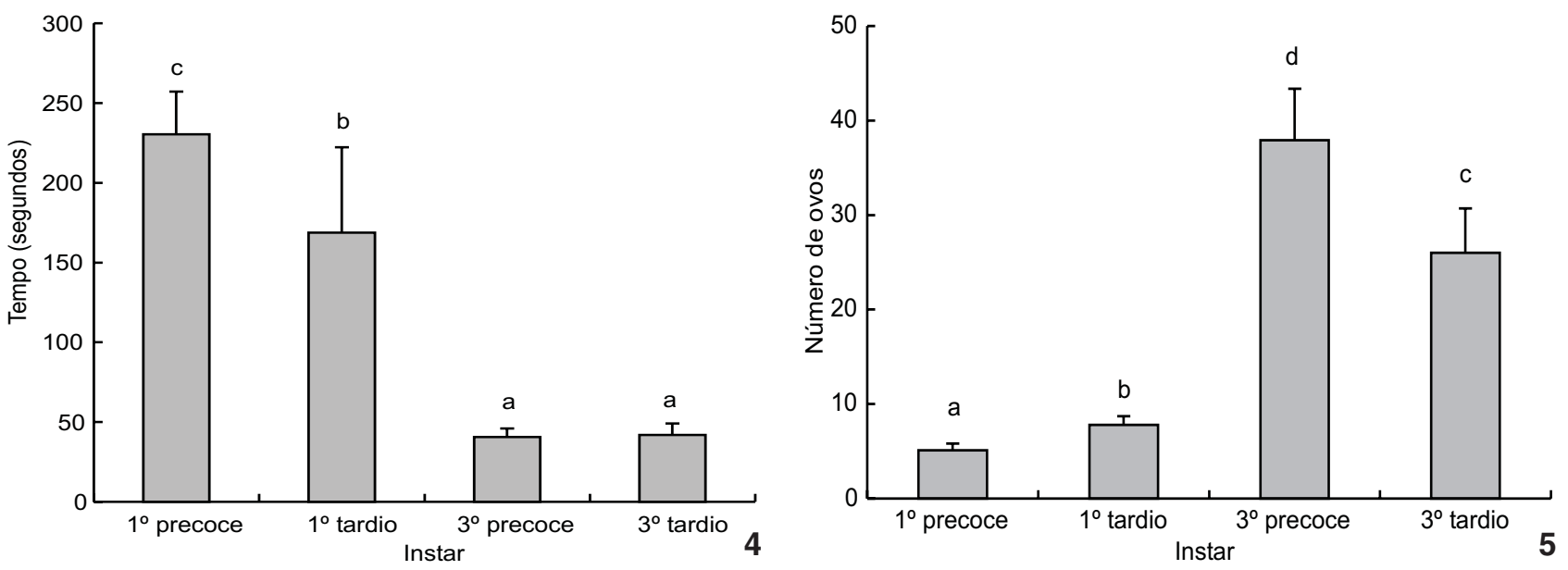

Figuras 4-5. (4) Tempo requerido pelas larvas de Leucochrysa varia para consumir um ovo de Anagasta kuehniella (média \pm EP; $\mathrm{n}=10$ larvas/instar/momento) (teste $\mathrm{t}, \mathrm{p}<0,01$ ); (5) número de ovos predados pelas larvas de Leucochrysa varia no período de 45 minutos de observação (média $\pm \mathrm{EP} ; \mathrm{n}=10$ larvas/instar/momento) (teste $\mathrm{t}, \mathrm{p}<0,01$ ).

Tabela III. Tempo despendido pelas larvas de Leucochrysa varia nas atividades comportamentais, em cada ocasião de sua exibição (média em segundos $\pm E P$, amplitude entre parênteses; $n=10$ larvas/instar/ocasião). (A) Significância das comparações de cada atividade comportamental entre idades no $1^{\circ}$ e no $3^{\circ}$ instar; (B) significância das comparações entre os instares. ${ }^{*}=p>0,05 ;{ }^{* *}=p>0,01 ;{ }^{* * *}=$ p > 0,001; NS = não significativo (ANOVA).

\begin{tabular}{|c|c|c|c|c|c|c|c|}
\hline \multirow{2}{*}{ Atividade } & \multicolumn{3}{|c|}{$1^{\circ}$ instar } & \multirow{2}{*}{ B } & \multicolumn{3}{|c|}{$3^{\circ}$ instar } \\
\hline & Precoce & A & Tardio & & Precoce & A & Tardio \\
\hline Alimentação & $\begin{array}{c}222,7 \pm 34,94 \\
(99-389)\end{array}$ & NS & $\begin{array}{c}274,2 \pm 48,18 \\
(87-588)\end{array}$ & $* * *$ & $\begin{array}{c}84,6 \pm 9,52 \\
(40-135)\end{array}$ & NS & $\begin{array}{c}71,0 \pm 14,45 \\
(37-196)\end{array}$ \\
\hline Imobilidade & $\begin{array}{c}174,2 \pm 49,35 \\
(25-429)\end{array}$ & NS & $\begin{array}{c}308,0 \pm 136,02 \\
(19-621)\end{array}$ & NS & $\begin{array}{c}178,3 \pm 35,37 \\
(57-408)\end{array}$ & NS & $\begin{array}{c}330,4 \pm 114,58 \\
(96-1254)\end{array}$ \\
\hline Mobilidade & $\begin{array}{c}93,1 \pm 18,74 \\
(29-198)\end{array}$ & NS & $\begin{array}{c}77,8 \pm 25,95 \\
(22-253)\end{array}$ & $* *$ & $\begin{array}{c}34,3 \pm 5,41 \\
(11-67)\end{array}$ & NS & $\begin{array}{c}62,6 \pm 15,01 \\
(17-136)\end{array}$ \\
\hline Camuflagem & $\begin{array}{c}49,0 \pm 10,38 \\
(18-103)\end{array}$ & NS & $\begin{array}{c}82,4 \pm 15,46 \\
(25-140)\end{array}$ & $* *$ & $\begin{array}{c}32,8 \pm 3,63 \\
(21-54)\end{array}$ & NS & $\begin{array}{c}28,7 \pm 4,50 \\
(17-60)\end{array}$ \\
\hline Limpeza & $\begin{array}{c}12,9 \pm 1,94 \\
(5-22)\end{array}$ & * & $\begin{array}{c}22,1 \pm 11,73 \\
(10-50)\end{array}$ & NS & $\begin{array}{c}17,7 \pm 1,24 \\
(12-26)\end{array}$ & NS & $\begin{array}{c}13,3 \pm 2,83 \\
(8-16)\end{array}$ \\
\hline
\end{tabular}




\section{Imobilidade}

As larvas de $1^{\circ}$ instar e as de $3^{\circ}$ precoces passaram cerca de $1 / 4$ de seu tempo paradas, em posição de repouso, enquanto este tempo aumentou drasticamente para as larvas de $3^{\circ}$ instar tardio, que passaram metade de seu tempo imóveis (Fig. 3, Tab. II). Em cada momento que o comportamento foi exibido, as larvas tardias ficaram quase o dobro do tempo paradas que as precoces em ambos instares ( cinco versus três minutos), embora estas diferenças não tenham sido significativas pela grande variabilidade entre as larvas observadas (Tab. III). As larvas tardias repetiram atividades de imobilidade em maior número que as precoces (Tab. II).

A partir do estado imóvel, as larvas realizaram qualquer uma das outras atividades, exceto o $1^{\circ}$ instar precoce, que não se camuflou após o repouso (Fig. 3). Estas priorizaram as atividades de mobilidade e alimentação na mesma proporção $(45,9 \%)$, enquanto as de $1^{\circ}$ instar tardio preferiram se camuflar $(35,1 \%)$, comer $(32,4 \%)$ ou se deslocar $(29,7 \%)$. Já o $3^{\circ}$ instar (precoce e tardio) preferiram se alimentar após o repouso (50 e $47,2 \%$, respectivamente).

\section{Mobilidade}

Esta categoria incluiu atividades de caminhada ou corrida executadas dentro da arena de observação. As larvas de $1^{\circ}$ instar precoce foram as mais ativas, despendendo 30\% de seu tempo em deslocamentos; a partir do $1^{\circ}$ instar tardio, houve uma redução significativa (12\%), que continuou no $3^{\circ}$ instar (8 e 7\% para precoce e tardio, respectivamente) (Fig. 3, Tab. II). Também houve redução no tempo gasto em cada vez que a larva caminhava, do $1^{\circ}$ para o $3^{\circ}$ instar (Tab. III). As larvas precoces repetiram atividades de mobilidade duas vezes mais que as tardias (Tab. II).

Quaisquer das demais atividades foram executadas após o deslocamento das larvas (Fig. 3). As larvas de $1^{\circ}$ instar precoce e ambas de $3^{\circ}$ instar priorizaram a alimentação $(34,6 ; 54,3$ e $52,9 \%$, respectivamente), enquanto as de $1^{\circ}$ instar tardias preferiram repousar $(45,7 \%)$.

\section{Camuflagem}

Para carregar os ovos de A. kuehniella, as larvas apreenderam e ergueram este material com as mandíbulas, curvando a cabeça para trás e arqueando o abdome, encaixando os ovos entre as longas cerdas que revestem o dorso do tórax e abdome, assim como os tubérculos laterais. Além disto, observou-se que, com exceção do $1^{\circ}$ instar precoce, as larvas realizaram movimentos com a extremidade do abdome em direção ao seu dorso nos intervalos de carregamento dos ovos, depositando gotículas de secreção adesiva nas cerdas e nos próprios ovos, que provavelmente servem para agregar o pacote de lixo.

As larvas despenderam sempre $20 \%$ ou menos de seu tempo na atividade de camuflagem (Fig. 3, Tab. II). As larvas tardias de $1^{\circ}$ instar foram as que despenderam tempo significativamente maior; nas demais, este tempo foi inferior a 10\% (Tab. II). Tendência semelhante foi observada em relação a cada oca- sião de camuflagem, pois o $1^{\circ}$ instar despendeu maior tempo cada vez que carregava material para seu dorso (Tab. III). As larvas tardias repetiram atividades de camuflagem em maior número que as precoces dos instares respectivos (Tab. II).

Após atos de camuflagem, quaisquer das quatro atividades foram observadas, com exceção do $1^{\circ}$ instar precoce, que não efetuou limpeza após camuflar-se (Fig. 3). As larvas de $1^{\circ}$ instar precoce priorizaram a mobilidade $(65,2 \%)$ após a camuflagem, enquanto as tardias e as de $3^{\circ}$ instar de ambas idades priorizaram a alimentação (40; 78,2 e 83,5\%, respectivamente).

\section{Limpeza}

Após sugar completamente o conteúdo interno dos ovos, as larvas removiam o cório restante de suas mandíbulas, esfregando-as uma contra a outra ou contra o substrato.

A limpeza foi sempre a atividade que menor tempo demandou das larvas (entre 1 e 4\%), independente do instar ou idade (Fig. 3, Tab. II). Apesar de pequenas, ainda assim houve diferença significativa nas proporções entre o $1^{\circ}$ e $3^{\circ}$ instar, resultante do tempo duas vezes superior despendido por larvas de $3^{\circ}$ instar precoce ao se limparem (Tab. II). O tempo médio despendido pelas larvas de $1^{\circ}$ instar tardio foi significativamente maior que o precoce em cada ocasião de limpeza, não tendo havido diferenças dentro do $3^{\circ}$ instar e entre os instares (Tab. III). Por outro lado, as larvas de $3^{\circ}$ instar repetiram a atividade de limpeza mais vezes que as de $1^{\circ}$ instar no período de 45 minutos (Tab. II).

Após cada ocasião de limpeza, as larvas realizaram qualquer uma das quatro outras atividades (Fig. 3). As larvas de $1^{\circ}$ instar precoce priorizaram em muito a mobilidade (76,5\%) após a limpeza, enquanto as tardias priorizaram a camuflagem $(42,9 \%)$. Em ambas idades do $3^{\circ}$ instar, o ato de limpar-se foi seguido principalmente por nova alimentação $(64,7$ e $71,4 \%$ para o precoce e tardio, respectivamente).

\section{Prioridade de COMPORTAMENTO (ALIMENTAÇÃO X CAMUFLA- GEM) E DURAÇÃO DO PRIMEIRO CARREGAMENTO}

A porcentagem de larvas que carregaram ovos para seu dorso antes de se alimentar foi consistente durante todo o desenvolvimento, isto é, 30-40\% (Tab. IV). Quanto à duração do primeiro ato de camuflagem, houve a tendência à diminuição no tempo gasto com o aumento da idade da larva, embora esta

Tabela IV. Prioridade de comportamento (alimentação x camuflagem) demonstrada pelas larvas de Leucochrysa varia frente à presa/material de camuflagem oferecido (ovos de Anagasta kuehniella) após o intervalo de 4-16 horas que permaneceram nuas e em jejum (\%, $\mathrm{n}=10$ larvas/instar/momento).

\begin{tabular}{lccccc}
\hline \multirow{2}{*}{ Comportamento } & \multicolumn{2}{c}{$1^{\circ}$ instar } & & \multicolumn{2}{c}{$3^{\circ}$ instar } \\
\cline { 2 - 3 } \cline { 5 - 6 } & Precoce & Tardio & & Precoce & Tardio \\
\hline Alimentação & 60,0 & 70,0 & & 60,0 & 70,0 \\
Camuflagem * & 40,0 & 30,0 & & 40,0 & 30,0 \\
\hline
\end{tabular}

* Ato de apreender um ovo de $A$. kuehniella (cheio ou vazio) com as mandíbulas e colocá-lo em seu dorso.

Revista Brasileira de Zoologia 24 (2): 302-311, junho 2007 
Tabela V. Duração do primeiro ato de camuflagem, em segundos, e número de ovos de Anagasta kuehniella carregados neste ato pelas larvas de Leucochrysa varia após o intervalo de 4-16 horas que permaneceram nuas e em jejum (média \pm EP, amplitude entre parênteses; $\mathrm{n}=10$ larvas/instar/momento).

\begin{tabular}{|c|c|c|c|c|}
\hline \multirow{2}{*}{ Camuflagem } & \multicolumn{2}{|c|}{$1^{\circ}$ instar * } & \multicolumn{2}{|c|}{$3^{\circ}$ instar } \\
\hline & Precoce & Tardio & Precoce & Tardio \\
\hline Duração & $41,4 \pm 9,74(8-103)$ & $40,3 \pm 6,79(11-74)$ & $35,7 \pm 4,65(21-67)$ & $32,3 \pm 6,31(9-60)$ \\
\hline $\mathrm{N}^{0}$ ovos carregados & $1,7 \pm 0,33(1-4)$ & $1,4 \pm 0,18(1-2)$ & $2,2 \pm 0,36(1-4)$ & $1,8 \pm 0,28(1-3)$ \\
\hline
\end{tabular}

* Uma larva carregou 24 ovos por 339 segundos $(P)$ e outra 10 ovos por 146 segundos $(T)$ ininterruptamente, valores estes desconsiderados para efeito de cálculo.

tenha sido reduzida (de 41 para 32 segundos) (Tab. V). O número de ovos carregados neste tempo também foi semelhante, em torno de dois ovos (Tab. V), embora uma larva de $1^{\circ}$ instar precoce tenha carregado 24 ovos em seqüência, por 339 segundos, enquanto outra de $1^{\circ}$ instar tardio tenha carregado 10 ovos por 146 segundos, valores que foram excluídos dos cálculos das médias, mas que demonstram a importância da atividade de camuflagem na vida das larvas, o que é corroborado pela prioridade dada à camuflagem antes da alimentação por quase metade das larvas.

\section{A CAMUFLAGEM E SUAS CONSEQÜÊNCIAS PARA O DESENVOLVIMENTO}

Os resultados obtidos para as larvas de L. varia assemelham-se mais aos obtidos para larvas de uma espécie generalista (Chrysopa quadripunctata Burmeister, 1839) do que para as de uma especialista (Chrysopa slossonae Banks, 1924), em estudo semelhante de Milbrath et al. (1993). Segundo estes autores, o especialista tenderia a priorizar mais a camuflagem do que a alimentação, devido ao fato de enfrentar seus inimigos naturais constantemente (formigas que cuidam da colônia de pulgões, suas presas); o comportamento críptico seria sua principal defesa. Por outro lado, o generalista tenderia a priorizar a alimentação, pois seu encontro com inimigos naturais é fortuito e ele usa a imobilidade como defesa principal. Por observações pessoais de campo e pelos resultados obtidos em laboratório, $L$. varia se enquadraria na categoria de generalista, pois suas larvas não foram encontradas em associação com nenhuma presa específica e o tempo que destinaram à imobilidade foi muito maior do que à camuflagem, principalmente no $3^{\circ}$ instar tardio.

Quanto às conseqüências do comportamento de carregamento de lixo para o desenvolvimento, não se encontraram evidências de que o número relativamente reduzido de cerdas de $L$. varia demande das larvas tempo demasiado para esta atividade, o que viria a comprometer sua capacidade de procura e ingestão de presas e, conseqüentemente, prolongaria seu ciclo de vida. O uso de gotículas de secreção adesiva para agrupar o material de camuflagem e formar o pacote de lixo, comportamento aparentemente desconhecido para larvas de crisopídeos, talvez tenha evoluído justamente para suprir a carência de cerdas no seu corpo, o que precisa ser testado. Até o presente, tais secreções, produzidas pelos túbulos de Malpighi e eliminadas pelo ânus das larvas, são associadas apenas às funções de adesão no substrato, durante sua locomoção (Killington 1936, Spiegler 1962), e de defesa, atuando como repelente contra seus inimigos naturais (LaMunyon \& Adams 1987, Canard \& Volkovich 2001). Portanto, o desenvolvimento longo de $L$. varia parece ser uma característica intrínseca da espécie, independente de seu comportamento de camuflagem, que estaria relacionado com o ambiente em que ocorre, onde as presas são escassas e dispersas.

\section{AGRADECIMENTOS}

À Catherine A. Tauber (Cornell University, EUA), pela confirmação da identidade de Leucochrysa (Leucochrysa) varia; a Edenio Detmann (UENF), pelo auxílio na análise estatística dos resultados do comportamento larval, a Jatinder S. Multani (UENF), pela ajuda nas observações do comportamento larval, a Ederaldo A. da Silva (UENF), pelo auxílio nas coletas de campo, e aos dois consultores anônimos, pela revisão crítica do manuscrito e sugestões de melhoria. À CAPES, pela bolsa de mestrado (EM) e ao CNPq, pela bolsa de produtividade em pesquisa (Processo 300504/96-9) e auxílio financeiro (Processo 466439/00-8) (GSA).

\section{REFERÊNCIAS BIBLIOGRÁFICAS}

Adams, P.A. 1987. Studies in Neotropical Chrysopidae (Neuroptera) III. Notes on Nodita amazonica Navás and N. oenops, sp. nov. Neuroptera International 4 (4): 287-294.

Adams, P.A. \& N.D. Penny. 1987. Neuroptera of the Amazon basin. Part IIa. Introduction and Chrysopini. Acta Amazonica 15 (3-4): 413-479.

Albuquerque, G.S.; C.A. Tauber \& M.J. Tauber. 1994. Chrysoperla externa (Neuroptera: Chrysopidae): life history and potential for biological control in Central and South America. Biological Control 4 (1): 8-13.

Brooks, S.J. \& P.C. Barnard. 1990. The green lacewings of the world: a generic review (Neuroptera: Chrysopidae). Bulletin of the British Museum of Natural History (Entomology) 59 (2): 117-286.

Canard, M. \& M.M. Principi. 1984. Development of Chrysopidae, p. 57-75. In: M. CANARd, Y. SÉméria \& T.R. New (Eds). Biology of Chrysopidae. The Hague, W. Junk Publishers, X+294p.

Canard, M. \& T.A. Volkovich. 2001. Outlines of lacewing development, p. 130-153. In: P.K. McEwen, T.R. New \& A.E.

Revista Brasileira de Zoologia 24 (2): 302-311, junho 2007 
Whittington (Eds). Lacewings in the crop environment. Cambridge, Cambridge University Press, XVIII+546p.

Díaz-Aranda, L.M.; V.J. Monserrat \& C.A. Tauber. 2001. Recognition of early stages of Chrysopidae, p. 60-81. In: P.K. McEwen, T.R. New \& A.E. Whittington (Eds). Lacewings in the crop environment. Cambridge, Cambridge University Press, XVIII+546p.

Freitas, S. \& N.D. Penny. 2001. The green lacewings (Neuroptera: Chrysopidae) of Brazilian agro-ecosystems. Proceedings of the California Academy of Sciences 52 (19): 245-395.

Gepp, J. 1984. Morphology and anatomy of the preimaginal stages of Chrysopidae: a short survey, p. 9-19. In: M. CANARD, Y. SÉméria \& T.R. New (Eds). Biology of Chrysopidae. The Hague, W. Junk Publishers, X+294p.

Killington, F.J. 1936. A monograph of the British Neuroptera. London, The Ray Society, vol. 1, XIX+269p.

LAMunyon, C.W. \& P.A. AdAms. 1987. Use and effect of an anal defensive secretion in larval Chrysopidae (Neuroptera). Annals of the Entomological Society of America 80 (6): 804-808.

Mantoanelli, E.; G.S. Albuquerque; C.A. TAuber \& M.J. Tauber. 2006. Leucochrysa (Leucochrysa) varia (Schneider) (Neuroptera: Chrysopidae): larval descriptions, developmental rates, and adult color variation. Annals of the Entomological Society of America 99 (1): 7-18.

Milbrath, L.R.; M.J. Tauber \& C.A. Tauber. 1993. Prey specificity in Chrysopa: an interspecific comparison of larval feeding and defensive behavior. Ecology 74 (5): 1384-1393.

Penny, N.D. 1977. Lista de Megaloptera, Neuroptera e Raphidioptera do México, América Central, Ilhas Caraíbas e América do Sul. Acta Amazonica 7 (4) (Supl.): 1-61.

Penny, N.D.; P.A. Adams \& L.A. Stange. 1997. Species catalog of the Neuroptera, Megaloptera, and Raphidioptera of America north of Mexico. Proceedings of the California Academy of Sciences 50 (3): 39-114.

Silva, P.S.; G.S. Albuquerque; C.A. Tauber \& M.J. Tauber. 2007. Life history of a widespread Neotropical predator, Chrysopodes (Chrysopodes) lineafrons (Neuroptera: Chrysopidae). Biological Control 41 (1): 33-41.

SLocum, R.D. \& J.D. LaWrey. 1976. Viability of the epizoic lichen flora carried and dispersed by green lacewing (Nodita pavida) larvae. Canadian Journal of Botany 54 (15): 1827-1831.

SPIEgler, P.E. 1962. The origin and nature of the adhesive substance in larvae of the genus Chrysopa (Neuroptera: Chrysopidae). Annals of the Entomological Society of America 55 (1): 69-77.

Tauber, C.A. 2003. Generic characteristics of Chrysopodes (Neuroptera: Chrysopidae), with new larval descriptions and a review of species from the United States and Canada. Annals of the Entomological Society of America 96 (4): 472-490.

Tauber, C.A. 2004. The genus Leucochrysa (Neuroptera: Chrysopidae) in the United States: a systematic review. Annals of the Entomological Society of America 97 (6): 1129-1158.

Tauber, C.A.; T. De León; N.D. Penny \& M.J. Tauber. 2000. The genus Ceraeochrysa (Neuroptera: Chrysopidae) of America north of Mexico: larvae, adults, and comparative biology. Annals of the Entomological Society of America 93 (6): 1195-1221.

Tauber, M.J. \& C.A. Tauber. 1976. Environmental control of univoltinism and its evolution in an insect species. Canadian Journal of Zoology 54 (2): 260-265.

WiggLesWorth, V.B. 1972. The principles of insect physiology. London, Chapman and Hall, $7^{\text {th }}$ ed., VIII+827p.

Recebido em 28.IV.2006; aceito em 28.IV.2007. 